\title{
COMPARISON OF THREE BIOCERAMIC SEALERS IN TERMS OF DENTINAL SEALING ABILITY IN THE ROOT CANAL
}

\section{SHALINA RICARDO ${ }^{1}$, CELINE MARISSA ${ }^{2}$, MUNYATI USMAN ${ }^{1}$, ENDANG SUPRASTIWII ${ }^{1}$, RENNA MAULANA YUSUF ${ }^{2}$, RATNA MEIDYAWATI ${ }^{*}$}

${ }^{1}$ Department of Conservative Dentistry, Faculty of Dentistry, Universitas Indonesia, Jakarta, Indonesia. ${ }^{2}$ Conservative Dentistry Residency Program, Faculty of Dentistry, Universitas Indonesia, Jakarta, Indonesia. Email: meidyawati58@gmail.com

Received: 30 July 2019, Revised and Accepted: 01 June 2020

ABSTRACT

Objective: The main objective of endodontic treatment is to prevent further infection or reinfection by eliminating microorganisms within the root canal system. Proper endodontic treatment could prevent apical and coronal penetration of fluids and microorganisms. Endodontic sealer is vital components of root canal obturation to establish a fluid-tight seal. Bioceramic-based root canal sealers are considered to be an advantageous technology in endodontics and have been found to be both biocompatible and comparable to other commercial sealers. The aim of this study was to compare the adhesion of three bioceramic sealers within the root canal system.

Methods: Endodontically treated teeth were obturated using three types of bioceramic sealers and then divided into three groups. Specimens were then observed using a scanning electron microscope, and the attachment distance was measured using ImageJ.

Results: The three groups exhibited were statistically significant differences $(\mathrm{p}<0.05)$ in dentinal sealing ability. Calcium phosphate silicate-based sealer showed the highest sealing ability, followed by pure tricalcium silicate-based bioceramic sealers and then tricalcium silicate and resin-based bioceramic sealers.

Conclusion: The sealing ability of calcium phosphate silicate-based sealer is superior to that of both pure tricalcium silicate-based and tricalcium silicate- and resin-based sealer.

Keywords: Bioceramics, Dentinal sealing ability, Sealers, Sealing ability, Dentinal tubules, Scanning electron microscope.

(C) 2020 The Authors. Published by Innovare Academic Sciences Pvt Ltd. This is an open access article under the CC BY license (http://creativecommons. org/licenses/by/4. 0/) DOI: http://dx.doi.org/10.22159/ijap.2020.v12s2.0P-6

\section{INTRODUCTION}

Root canal treatment performed to eliminate microorganisms and prevent reinfection. After cleaning and shaping, an effective root canal filling is necessary to maintain microorganism-free environment within the root canal and avoid recontamination [1-3]. The sealing ability, biocompatibility, and antimicrobial properties of root canal filling materials are important factors in accomplishing this task. Sealers that could adapt closely to the dentinal canal walls aimed at preventing leakage in the apical region [3-5]. As incomplete sealing of the root canals will lead to major endodontic failure, it is essential to use materials that can form a hermetic seal within the root canal system. Filling materials that evoke a biological response at the material dentin interface represent an improvement in the quality of sealing [6-9]

Bioactive endodontic sealers have been developed to improve the quality of root canal obturation. There are now three types of bioceramic sealers with different base materials. These sealers are calcium phosphate silicate-based bioceramic sealers (BioRoot RCS; Septodont, Saint-Maur-Des-Fosses, France), tricalcium silicate- and resin-based bioceramic sealers (MTA Fillapex; Angelus Industry Dental Products S/A, Londrina, PR, Brazil), and pure tricalcium silicate-based bioceramic sealers (iRoot; Innovative BioCeramix, Vancouver, BC, Canada) [10,11].

The components of bioceramic sealers are zirconium oxide, calcium silicates, calcium phosphate monobasic, calcium, hydroxide, fillers, and thickening agents. Contemporary studies on bioceramic-based sealer have found adequate characteristics, including its adhesive property [5-11].
The bioactive property of the sealer for obturation material, specifically certain compositions of glasses comprising $\mathrm{SiO}_{2}, \mathrm{CaO}, \mathrm{Na}_{2} \mathrm{O}$, and $\mathrm{P}_{2} \mathrm{O}_{5}$, can bond to either the dentinal or root tissue. In general, when the bioactive sealer comes into contact with the root canal, it may induce a phosphate buffered solution, forming a tag-like structure, and it may form a fluid-tight seal with the root canal. Bioceramic materials contain calcium phosphate, which enhances the setting properties of bioceramics and creates a chemical composition and crystalline structure similar to those of tooth and bone apatite materials [12-14], thus improving sealer-to-root dentin bonding.

This in vitro study was performed to assess and compare the apical sealing ability of three bioceramic-based sealers: Calcium phosphate silicate-based bioceramic sealers (BioRoot), tricalcium silicate- and resin-based bioceramic sealers (MTAF), and pure tricalcium silicatebased bioceramic sealers (iRoot), which are considered to be the gold standard of sealing and adhesion to dentin [15-17].

The aim of this study was to compare three bioceramic sealers in terms of sealer adhesion in the root canal. The bioceramic sealer may have similar dentinal sealing ability within the root canal, and better sealing ability could be obtained from the tested sealers.

\section{METHODS}

In this study, we used 27 single-rooted, human mandibular premolars that were cleaned with a scaler and soaked with $0.9 \% \mathrm{NaCl}$ solution. The experiment was performed under ethical clearance protocol number 051111018 from Universitas Indonesia Ethics and Research Department. Three team members who had been calibrated performed the experiments. Specimens were examined under an operating 
microscope (Carl Zeiss Meditec AG, Germany) at ×20, and we excluded teeth that were either fractured or cracked and had calcifications, resorbed roots, curved canals, or caries. Radiograph images (Digora, Soredex, Finland) were obtained to confirm the presence of a single unmanipulated canal without resorption and calcification. Specimens were accessed using round diamond burs and then prepared using the crown-down technique. A \#10 K-file (Dentsply Maillefer, Switzerland) was inserted into the root canal to the tip of the apex, and then, $0.5 \mathrm{~mm}$ was deducted from the apex; this length was determined as the working length. The samples were prepared through the crown-down technique using the Rotary ProTaper Next instrument (Dentsply Maillefer, Switzerland) until $\times 3$ with 0.07 taper.

EDTA gel with $17 \%$ concentration was used for preparation. Irrigation was conducted using $2 \mathrm{ml}$ of $2.5 \% \mathrm{NaOCl}$ and $1 \mathrm{ml} 17 \%$ EDTA between each instrumentation, and agitation was performed using sonic instruments (Endoactivator, Dentsply, Switzerland). Irrigation was then concluded using $2 \mathrm{ml}$ of distilled water.

Specimens that had been prepared were then obturated with guttapercha ProTaper Next cone (Dentsply Maillefer, Ballaigues, Switzerland) and bioceramic sealers (iRoot ${ }^{\circledR}$ SP, MTA Fillapex, and BioRoot RCS). Sealers were inserted into the root canal as per the manufacturer's protocols. The coronal area was sealed using resin-modified glass ionomer cement. Specimens then stored in an incubator for 5 days at $37^{\circ} \mathrm{C}$ and $100 \%$ humidity to allow the sealers to set.

The specimens were then mounted in a wax block, and the quality of root canal filling was assessed further using Digital Radiograph Imaging (Digora, Soredex, Finland). Roots were grooved longitudinally and split into two halves by placing a cement spatula in the grooves and applying gentle pressure. We then vacuum dried the sections, coated them with $20 \mathrm{~nm}$ gold, and examined them at the apical third of the root canal using a scanning electron microscope (SEM) (Carl Zeiss NTS GmbH, Oberkochen, Germany). We examined the dentinal sealing ability and adaptation of each sealant to the dentin at $\times 1500$ and then took microphotographs. These were then calculated with ImageJ software (LOCI, Wisconsin, USA).

The gap distances between filler material gutta-percha and dentinal tubules were measured by picking spots at random at the apical third of the cross-section. The data obtained were analyzed through parametric statistical tests using SPSS software ver. 22.0. Statistical analysis was conducted after first normality tests data. As the data distribution was normal (parametric), one-way ANOVA statistical test with a significance limit of $\alpha=0.05$ was performed.

\section{RESULTS AND DISCUSSION}

\section{SEM analysis}

SEM analyses of the root canals that were obturated using three different sealers showed that their adaptation to dentin was sufficient for good sealing ability along the root canal as seen at Fig 1 . The teeth filled with calcium silicate-based sealers exhibited a smooth contact line on the sealer-dentin interface as seen at Fig 2. There was a clear margin between the sealer and the dentinal walls. The teeth filled with guttapercha exhibited good adhesion to the dentinal walls (Figs 3 and 4), and clear bonding surfaces were observed. Moreover, the textures of the sealers along the root canal were homogenous. The finding was assessed by studying the gap distance between the sealer and the root canal.

\section{Statistic analysis}

One-way ANOVA confirmed the existence of significant differences in the dentinal sealing abilities of the three groups. Calcium phosphate silicate-based bioceramic sealers (BioRoot) was significantly has higher sealing ability than tricalcium silicate- and resin-based bioceramic sealers (MTAF) but showed no significant differences with pure tricalcium silicate-based bioceramic sealers (iRoot). The differences between the three groups were assessed using three independent samples through one-way ANOVA with a confidence interval of 95\%. SPSS statistical software (ver. 20.0, Chicago, IL) was used for all analyses, and $\mathrm{p}<0.05$ was considered statistically significant. Statistical analysis from Table 1 shows a significance value of $p=0.001(p<0.05)$, which reveals that there are significant differences in dentinal sealing ability between the three groups. The table also shows that Group 1 has the better sealing ability, followed by Group 3 and, last, Group 2 (Table 1).

Based on the significance value of a post hoc test (multicomparison analysis), the results of the homogeneity test were $\mathrm{p}<0.018$, with a significance limit of $<0.05$. As the data were heterogeneous, the post hoc.

Tamhane test was used. The test showed significant differences in the gap distance between the three bioceramic sealer groups, with a significance limit of $\mathrm{p}=0.001(\mathrm{p}<0.05)$ (Table 2$)$.

Several studies have demonstrated the presence of microorganisms, including bacteria, in the dentinal tubules and cementum following endodontic treatment [7-9]. A root canal sealer with high antimicrobial activity is an important tool for reducing the growth of microbes and preventing them from reentering the root canal system [10-13].

Bioceramic material is an ideal bioactive sealant, and available bioceramic sealers include three different core materials. These sealers are calcium phosphate silicate-based bioceramic sealers (BioRoot), tricalcium silicate- and resin-based bioceramic sealers (MTAF), and pure tricalcium silicate-based bioceramic sealers (iRoot). This study was conducted to compare the dentinal sealing abilities of these three sealers.

Root canal sealer adhesion defines as the capacity to aggregate the root filling material and maintains the filler as a compact mass along the root canal and provides a single block configuration that seals the canal space hermetically. Tagger et al. [18] argued that the term adhesion should be replaced with the term bonding when discussing sealers as the attachment between the substances involves mechanical interlocking forces and not molecular attraction. The potential adhesion of root canal filling materials has been tested commonly from the perspectives of bond strength and microleakage, and there have been no standard methods to measure it.

In a similar study, Zhang et al. (2009) examined the sealing abilities of bioceramic sealers and pure resin-based sealers. They found that both sealers produced gap-free and gap-containing regions within the root canals. In this study, we found SEM observation showed that the apical third adaptation of both bioceramic sealers is quite the same $[9,10]$. Another study demonstrated that removal of the smear layer had no

Table 1: The mean value and standard deviation of the dentinal sealing abilities of three different sealers

\begin{tabular}{llll}
\hline Sealer & n & Mean (SD) & p value \\
\hline Group 1 & 9 & $3.90(2.46)$ & 0.01 \\
Group 2 & 9 & $42.24(9.54)$ & \\
Group 3 & 9 & $4.99(2.17)$ & \\
\hline
\end{tabular}

Group 1 - calcium phosphate silicate-based bioceramic sealers (BioRoot),

Group 2 - tricalcium silicate- and resin-based bioceramic sealers (MTA Fillapex), Group 3 - pure tricalcium silicate-based bioceramic sealers (iRoot)

Table 2: The significance values of the dentinal sealing ability of three different sealers

\begin{tabular}{llll}
\hline Sealer type & Group 1 & Group 2 & Group 3 \\
\hline Group 1 & $0.001^{*}$ & 0.708 \\
Group 2 & & $0.001^{*}$ \\
\hline
\end{tabular}

Post hoc Tamhane test, $\mathrm{p}<0.05$ 

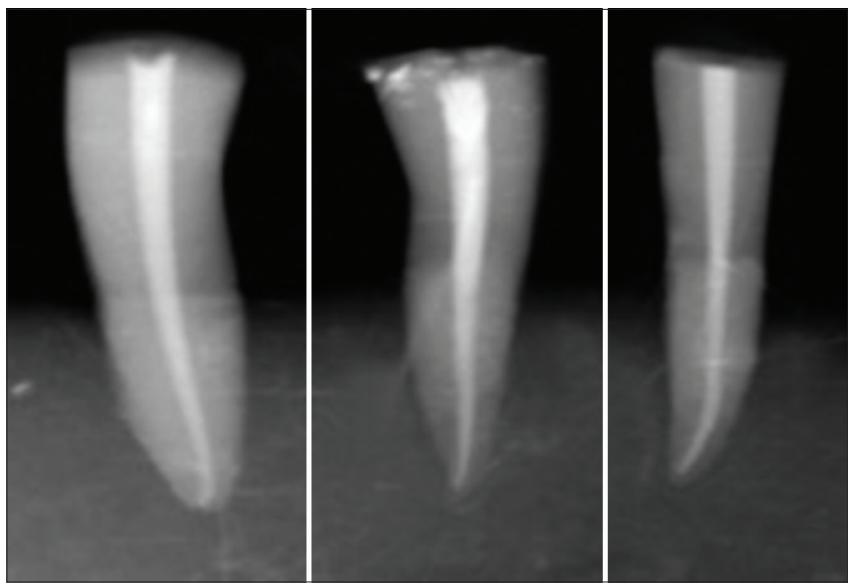

Fig. 1: Digital radiograph evaluation of obturation. A. Group 1: Calcium phosphate silicate-based bioceramic sealer. B, Group 2: Tricalcium silicate- and resin-based bioceramic sealer. C. Group 3: Pure tricalcium silicate-based bioceramic sealer

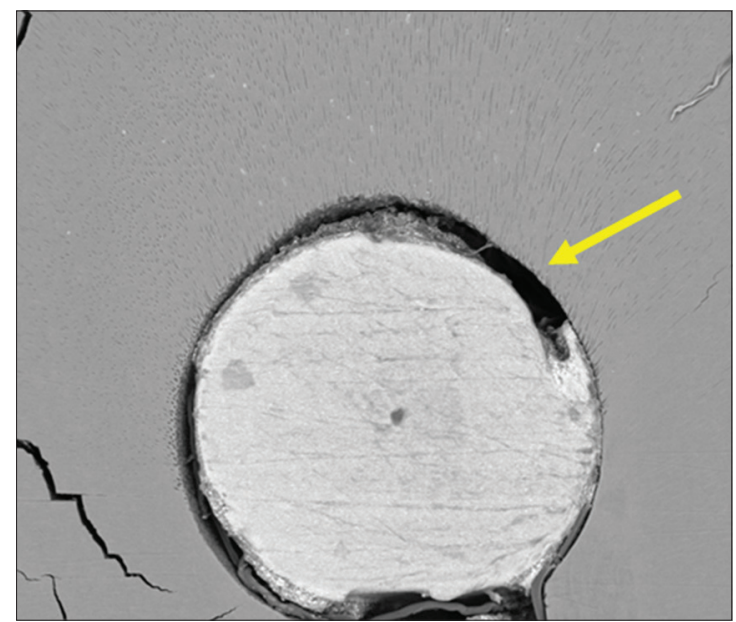

Fig. 2: Samples of Group 2 shows a mild gap between the sealer and the root canal

direct effect on the sealing ability of pure tricalcium silicate-based sealer, but the sealing ability with resin addition was less than was that of pure tricalcium silicate $[18,19]$. The radiopacity (equal to $7.06 \mathrm{~mm}$ of aluminum) and flow of bioceramic sealers were higher than were those of resin added sealers.

Solubility is related to the sealing ability of a sealer, as it must be bonded tightly to dentin and to root canal filling cones $[4,7,15,16]$. Several studies have assessed the sealing abilities of different bioceramicbased sealers in vitro. Further, various methodologies have revealed that the sealing ability of bioceramic-based sealers is satisfactory and comparable to that of other commercially available sealers. We used the same methodology to compare three different sealers to determine their relative performances to achieve a better prognosis in treating patients [17,20, 21-23].

The morphology of the root canal may influence the quality of obturation. We used mandibular premolars with single and straight canals to simplify the preparation process and facilitate operation of the microscope. Irrigation solutions of $2 \mathrm{ml}$ each of $2.5 \% \mathrm{NaOCl}$ and $17 \%$ EDTA were applied to clean the root canal effectively, remove the smear layer, and open the dentinal tubules. EDTA also has low surface tension and could increase the porosity in root canal treatment, removing smear layers in particular, which could enhance the sealants' access and adaptation to the dentin.

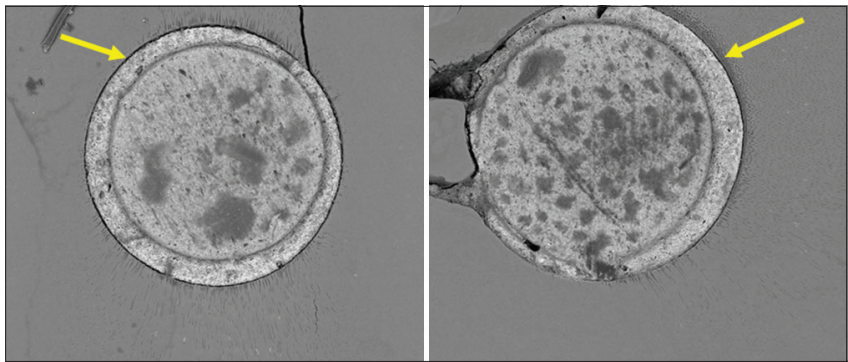

Fig. 3: Samples of Group 1 show the capability of the sealer to create intact seals of the root canal

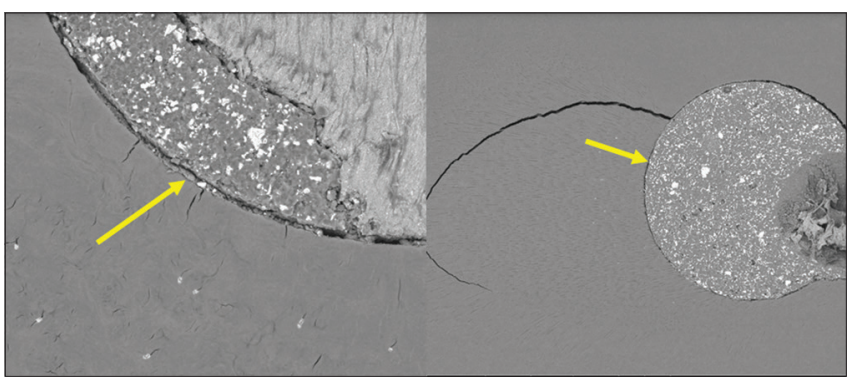

Fig. 4: Samples of Group 3 show some mild gaps in a couple of the dentinal walls and intact sealing ability for the rest of the canals

Our laboratory experiment compared the dentinal sealing ability of three different bioceramic sealers. Federer's formula was used to obtain a total sample of 27 teeth, divided into three groups with nine teeth in each group. We measured and calculated the gap between gutta-percha and the pulpodentinal junction $[13,24-26]$.

In this study, the teeth were cut with diamond disc until the cementenamel junction was reached to obtain a uniform working length of $15 \mathrm{~mm}$. Root canal preparation was performed using rotary instruments because that is the most common approach currently, and it simplifies the preparation process and provides a more uniform preparation.

SEM assesses the dentinal sealing ability and adhesiveness of the sealer to dentin walls at various levels of sectioning. In this study, SEM showed that specimens obturated with BioRoot and iRoot had good adhesion to the dentinal walls and to the gutta-percha core. As expected, there were differences in the adhesive properties of endodontic sealers because of their chemical and physical composition [18,19,27-29].

Testing the sealing ability at the root canal revealed no significant differences between calcium phosphate silicate-based bioceramic sealers and pure tricalcium silicate-based bioceramic sealers; however, it revealed significant differences with tricalcium silicateand resin-based bioceramic sealers. The tricalcium silicate structure has substantial granule, it would give adequate sealing ability in dentinal tubules, despite being lower than the other bioceramicbased sealers.

Within the limitations of this study, BioRoot and iRoot bioceramic sealers, along with gutta-percha, outperformed the gold standard, and the performance of MTAF was not up to par. However, further clinical studies are necessary to determine the long-term results with these materials, as it is better to have no gap at all, given that bacteria can be smaller than 4 microns.

\section{CONCLUSION}

Our findings indicated that bioceramic-based root canal sealers yield promising results and that calcium phosphate silicate-based bioceramic sealers (BioRoot) have better sealing ability than do pure tricalcium silicate-based bioceramic sealers and tricalcium silicate- and resin- 
based bioceramic sealers. Difference in the sealing ability of calcium phosphate silicate-based bioceramic sealers and pure tricalcium silicate-based bioceramic sealers was statistically insignificant, showing that the two groups have equal capacity.

\section{ACKNOWLEDGMENT}

This study was supported by the Directorate of Research and Community Engagement of Universitas Indonesia. The publication of this manuscript was supported by Universitas Indonesia. We thank Dwi Kamaratih Rubianto for assistance in paper writing.

\section{REFERENCES}

1. Schilder H. filling root canals in three dimensions 1967. J Endod 2006;32:281-90.

2. Sahebi S, Sobhnamayan F, Naghizade S. The effects of various endodontic irrigants on the push-out bond strength of calciumenriched mixture cement and mineral trioxide aggregate. Iran Endod J 2016;11:280-5

3. Carvalho CN, Bauer J, Ferrari PH, Souza SF, Soares SP, Loguercio AD, et al. Influence of calcium hydroxide intracanal medication on bond strength of two endodontic resin-based sealers assessed by micropushout test. Dent Traumatol 2013;29:73-6.

4. Desai S, Chandler N. Calcium hydroxide-based root canal sealers: A review. J Endod 2009;5:475-80.

5. Ozkocak I, Sonat B. Evaluation of effects on the adhesion of various root canal sealers after Er:YAG laser and irrigants are used on the dentin surface. J Endod 2015:41:1331-6

6. Neelakantan P, Subbarao C, Subbarao CV, De-Deus G, Zehnder M. The impact of root dentine conditioning on sealing ability and push-out bond strength of an epoxy resin root canal sealer. Int Endod J 2011;44:491-8.

7. Pawar AM, Pawar S, Kfir A, Pawar M, Kokate S. Push-out bond strength of root fillings made with C-point and $\mathrm{BC}$ sealer versus guttapercha and $\mathrm{AH}$ plus after the instrumentation of oval canals with the self-adjusting file versus WaveOne. Int Endod J 2016;49:374-81.

8. Chivian N. Resilon-the missing link in sealing the root canal. Compend Contin Educ Dent 2004;25:823.

9. Singh H, Markan S, Kaur M, Gupta G. Endodontic sealers: Current concepts and comparative analysis. Dent Open J 2015;2:32-7.

10. Gutmann JL. Adaptation of injected thermoplasticized gutta-percha in the absence of the dentinal smear layer. Int Endod J 1993;26:87-92

11. Chen H, Zhao X, Qiu Y, Xu D, Cui L, Wu B. The tubular penetration depth and adaption of four sealers: A scanning electron microscopic study. Biomed Res Int 2017;2017:2946524.

12. Camilleri J. BioRoot ${ }^{\mathrm{TM}}$ RCS. Endo Sealer or Biological Filler? Milano: Clinical Insights; 2017.

13. Siboni F, Taddei P, Zamparini F, Prati C, Gandolfi MG. Properties of
BioRoot RCS, a tricalcium silicate endodontic sealer modified with povidone and polycarboxylate. Int Endod J 2017;50 suppl 2:e120-36.

14. Zhou HM, Shen Y, Wang ZJ, Li L, Zheng YF, Häkkinen L, et al. In vitro cytotoxicity evaluation of a novel root repair material. J Endod 2013;39:478-83

15. Abada HM, Farag AM, Alhadainy HA, Darrag AM. Push-out bond strength of different root canal obturation systems to root canal dentin. Tanta Dent J 2015;12:185-91.

16. Sagsen B, Ustün Y, Demirbuga S, Pala K. Push-out bond strength of two new calcium silicate-based endodontic sealers to root canal dentine. Int Endod J 2011;44:1088-91.

17. McMichael GE, Primus CM, Opperman LA. Dentinal tubule penetration of tricalcium silicate sealers. J Endod 2016;42:632-6.

18. Tagger M, Tagger E, Tjan AH, Bakland LK. Measurement of adhesion of endodontic sealers to dentin. J Endod 2002;28:351-4.

19. Kim JS, Baek SH, Bae KS. in vivo study on the biocompatibility of newly developed calcium phosphate-based root canal sealers. J Endod 2004;30:708-11

20. Mamootil K, Messer HH. Penetration of dentinal tubules by endodontic sealer cements in extracted teeth and in vivo. Int Endod J 2007:40:873-81.

21. Zhang W, Li Z, Peng B. Assessment of a new root canal sealer's apical sealing ability. Oral Surg Med Oral Pathol Oral Radiol Endod 2009;107:e79-82.

22. Ghoneim AG, Lutfy RA, Sabet NE, Fayyad DM. Resistance to fracture of roots obturated with novel canal-filling systems. J Endod 2011;37:1590-2.

23. Chandra SS, Shankar P, Indira R. Depth of penetration of four resin sealers into radicular dentinal tubules: A confocal microscopic study. J Endod 2012;38:1412-6.

24. Tuncer AK, Tuncer S, Gökyay SS. Correlation between sealer penetration into dentinal tubules and bond strength of two new calcium silicate-based and an epoxy resin-based, endodontic sealer. J Adhes Sci Technol 2014:28:702-10

25. Camilleri J, Sorrentino F, Damidot D. Characterization of un-hydrated and hydrated BioAggregate ${ }^{\mathrm{TM}}$ and MTA Angelus ${ }^{\mathrm{TM}}$. Clin Oral Investig 2015;19:689-98

26. Leal F, De-Deus G, Brandao C, Luna A, Souza E, Fidel S. Similar sealability between bioceramic putty ready-to-use repair cement and white MTA. Braz Dent J 2013;24:362-6.

27. Yoshikawa M, Hayami S, Tsuji I, Toda T. Histopathological study of a newly developed root canal sealer containing tetracalcium-dicalcium phosphates and 1.0\% chondroitin sulfate. J Endod 1997;23:162-6.

28. Bilginer S, Esener IT, Söylemezoğlu F, Tiftik AM. The investigation of biocompatibility and apical microleakage of tricalcium phosphate based root canal sealers. J Endod 1997;23:105-9.

29. Zoufan K, Jiang J, Komabayashi T, Wang YH, Safavi KE, Zhu Q. Cytotoxicity evaluation of gutta flow and endo sequence BC sealers. Oral Surg Oral Med Oral Pathol Oral Radiol Endod 2011;112:657-61. 